Article

\title{
New BFS Retrieval Technique for Brillouin Optical Time Domain Analysis Sensor System
}

\author{
Haoyu Wei ${ }^{1,+}$, Yongjun Wang ${ }^{1, *}$, Qiming Wang ${ }^{1,+}$, Xiya Lu ${ }^{1,+}$, Hongxin $\mathrm{Wu}^{2,+}$, Lei Fan ${ }^{1,+}$, Chao Li ${ }^{1,+}$ \\ and Xiangjun Xin ${ }^{1,+}$ \\ 1 State Key Laboratory of Information Photonics and Optical Communications, School of Electronic \\ Engineering, Beijing University of Posts and Telecommunications, Xitucheng Road NO. 10, \\ Beijing 100876, China; weihaoyu@bupt.edu.cn (H.W.); clear@bupt.edu.cn (Q.W.); \\ lalalawyt@bupt.edu.cn (X.L.); xuhui2017@bupt.edu.cn (L.F.); lythao@bupt.edu.cn (C.L.); \\ xjxin@bupt.edu.cn (X.X.) \\ 2 International School, Beijing University of Posts and Telecommunications, Xitucheng Road NO. 10, \\ Beijing 100876, China; wuhongxin@bupt.edu.cn \\ * Correspondence: wangyj@bupt.edu.cn; Tel.: +86-18611360771 \\ + These authors contributed equally to this work.
}

check for updates

Citation: Wei, H.; Wang, Y.; Wang, Q.; Lu, X.; Wu, H.; Fan, L.; Li, C.; Xin, $X$. New BFS Retrieval Technique for Brillouin Optical Time Domain Analysis Sensor System. Electronics 2021, 10, 1334. https://doi.org/ 10.3390/electronics10111334

Academic Editor: Martin Reisslein

Received: 26 April 2021

Accepted: 29 May 2021

Published: 2 June 2021

Publisher's Note: MDPI stays neutral with regard to jurisdictional claims in published maps and institutional affiliations.

Copyright: (c) 2021 by the authors. Licensee MDPI, Basel, Switzerland. This article is an open access article distributed under the terms and conditions of the Creative Commons Attribution (CC BY) license (https:// creativecommons.org/licenses/by/ $4.0 /)$.

\begin{abstract}
In this paper, Gaussian smoothing (GS), non-local means (NLM), and Quaternion Wavelet Transform (QWT) have been described in detail. Furthermore, a Brillouin optical time domain analysis (BOTDA) experimental system was built to verify the denoising algorithms. The principal and experimental analyses show that the QWT algorithm is a more efficient image denoising method. The results indicate that the GS algorithm can obtain the highest signal-to-noise ratio (SNR), frequency uncertainty, and Brillouin frequency shift (BFS) accuracy, and can be executed in an imperceptible time, but the GS algorithm has the lowest spatial resolution. After being denoised by using NLM algorithm, although SNR, frequency uncertainty, BFS accuracy, and spatial resolution significantly improved, it takes $40 \mathrm{~min}$ to implement the NLM denoising algorithm for a BGS image with $200 \times 100,000$ points. Processed by the QWT denoising algorithm, although SNR increases to $17.26 \mathrm{~dB}$ and frequency uncertainty decreases to $0.24 \mathrm{MHz}$, a BFS accuracy of only $0.2 \mathrm{MHz}$ can be achieved. Moreover, the spatial resolution is $3 \mathrm{~m}$, which is not affected by the QWT denoising algorithm. It takes less than $32 \mathrm{~s}$ to denoise the same raw BGS data. The QWT image denoising technique is suitable for BGS data processing in the BOTDA sensor system.
\end{abstract}

Keywords: distributed sensing; Brillouin optical time-domain analysis; quaternion wavelet transform; Brillouin frequency shift retrieval

\section{Introduction}

Thermal-induced acoustic waves (acoustic phonons) produce periodic modulation of a refractive index of silica-based optical fibers (a moving refractive index grating) via an electrostriction process and travel along the fiber at a speed of acoustic velocity determined by the fiber parameters, such as Young's modulus, Poisson ratio, and density, which are influenced by some environment parameters. When pumping, a light pulse propagates along the fiber in the same direction as this moving refractive index grating and Brillouin scattering is induced by pump light diffracted backward by this moving refractive index grating. Brillouin scattering gives rise to downshifted frequency waves whose frequency shift depends on the acoustic velocity. In Brillouin optical time domain analysis (BOTDA), a continuous wave probe light is injected from the opposite end of the fiber and interacts with the pump pulses in the fiber. If the frequency difference between the pump and probe beam is close to the local Brillouin frequency shift (BFS), the pump light will convert part of its energy to the probe light. BOTDA is suitable for long-distance temperature and stress measurement by its high detection power. Theoretical and experimental results 
show that the change of local BFS, being retrieved by the central frequency of Brillouin gain spectrum (BGS), is proportional to the temperature or strain change $[1,2]$. Due to its capability of acquisition of a spatially distributed temperature, strain, and pressure, the BOTDA sensing system has promise to be widely applied in structural health monitoring in some major infrastructure, such as in airport fences, oil pipelines, hydroelectric dams, submarine optical fiber cables, and so on [3,4]. In general, the BFS distribution can be obtained from the BGS profile by sweeping the frequency of probe light and detecting the intensity gains of the probe signal. The raw BGS data being often drowned in severe noise, it is difficult to achieve accurate BFSs from these noisy BGS curves even if the collected BGS data are averaged hundreds of times to eliminate the random noise [5-7]. A real-time BOTDA measurement system requires that the data acquisition time is reduced to seconds or even milliseconds, and it is expected to average dozens of times. Therefore, in order to meet the requirements of high precision and high spatial resolution real-time measurement, one of researchers' main tasks is focused on finding some highly time-efficient methods to improve the signal-to-noise ratio (SNR) of BGS. In recent years, some conventional opticaldomain signal process techniques, such as many optical pulse coding $[8,9]$ or pump pulse improving [10], have been proposed to reduce noise in BGS data or suppress pump pulse distortion, but these techniques usually results in complex optical processing or additional optical device investment. Although with the development of digital signal processing technology, many digital denoising methods, such as wavelet denoising (WD) [11] and the lifting wavelet transform (LWT) [12], have been used for BGS denoising and can be implemented with high time efficiency, these traditional algorithms have limited denoising effects. Because BGS can be mapped into a 2-dimensional (2D) or 3D image, using mature image processing technology to denoise BGS data has become the focus of current research. Up to now, various image denoising methods, such as 2D wavelet denoising (2D-WD), non-local means (NLM) [13], block matching 3D filter (BM3D) [14], image denoising based on convolutional neural network (CNN) [15], and so on, have been introduced to BGS image denoising and remarkable SNR enhancement has been demonstrated experimentally. However, many image denoising algorithms have high computational complexity, and it often takes a long time to complete the image processing under the condition of good hardware and software configuration. In addition, many image denoising algorithms will give rise to the loss of useful details or blur the image, which will degrade the spatial resolution of BOTDA. Therefore, the application of many image denoising techniques in real-time monitoring is limited due to the poor time efficiency and low spatial resolution.

In order to enhance the time efficiency, improve measurement accuracy, and eliminate the degradation of spatial resolution from the image blurring, our research team proposed applying a Quaternion Wavelet Transform (QWT) image denoising algorithm to BGS image processing. In this paper, we reveal the algorithm principle of the QWT image denoising technique; list two image denoising algorithms-NLM and Gaussian smoothing (GS) filter algorithms - for comparison with the QWT denoising algorithm; and study their denoising effect and execution efficiency. The results indicate that QWT image algorithm not only has a good denoising effect, but also does not bring about a significant reduction in spatial resolution. Moreover, the efficiency of the algorithm can meet the requirements of many large infrastructures structure health monitoring. The research results of this paper have a certain guiding role for the application of QWT image denoising algorithm technique in BOTDA sensor system.

\section{BGS Image Denoising Algorithms}

The BGS image processed by denoising algorithms is mapped from raw BGS data which comes from a BOTDA experimental system with approximately $40 \mathrm{~km}$ long sensing fiber under test (FUT) shown in Figure 1a,b, which also shows the physical picture of our experimental system. A continuous wave (CW) semiconductor laser emits a laser beam with a $1550 \mathrm{~nm}$ wavelength and $10 \mathrm{kHz}$ line-width. The beam is split into two parts by a 50/50 optical coupler. One half of the upper arm as probe light is injected 
into a high-extinction ratio (>40 dB) Electro-optic modulator 1 (EOM1) and the other half of the lower arm as pump light is injected into EOM2. EOM1 is driven by a frequency synthesizer which can multiply a $100 \mathrm{MHz}$ clock signal to about $11 \mathrm{GHz}$ and change its output frequency according to the control command set through a universal serial interface. In our system, the output radio frequency signal from frequency synthesizer varies its frequency from $10.77 \mathrm{GHz}$ to $10.96 \mathrm{GHz}$ at a $1 \mathrm{MHz}$ step. EOM2 is controlled by a programmable pattern generator (PPG) which can generate pulse sequence with $30 \mathrm{~ns}$ time width and $4 \mathrm{kHz}$ repetition rate. The probe light is amplified by an erbium-doped fiber amplifier 1 (EDFA1), and its power is adjusted to about $8 \mathrm{dBm}$ at the beginning of FUT by variable optical attenuator (VOA). The EDFA2 is used to amplify pump pulse to achieve the peak power up to $20 \mathrm{dBm}$, while an optical band-pass filter (BPF) is used to filter out the amplified spontaneous emission noise. The pump pulses are launched into the other end of FUT through an optical circulator and counter-propagated with CW probe light along the sensing fiber. At the end of FUT, a section of about $200 \mathrm{~m}$ is put into an incubator, while the remainder of the fiber is kept at room temperature. Finally, the probe light is detected by a $125 \mathrm{MHz}$ high-transimpedance photodetector and collected by an oscilloscope (OSC). The collected results are averaged according to the required times and saved in the OSC's memory.

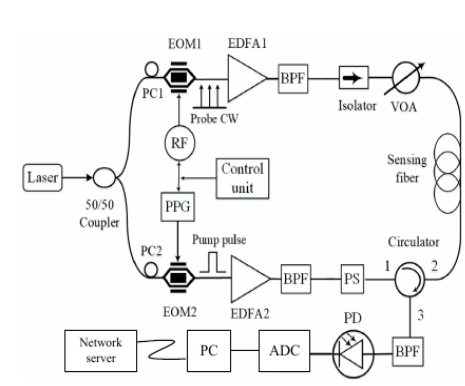

(a)

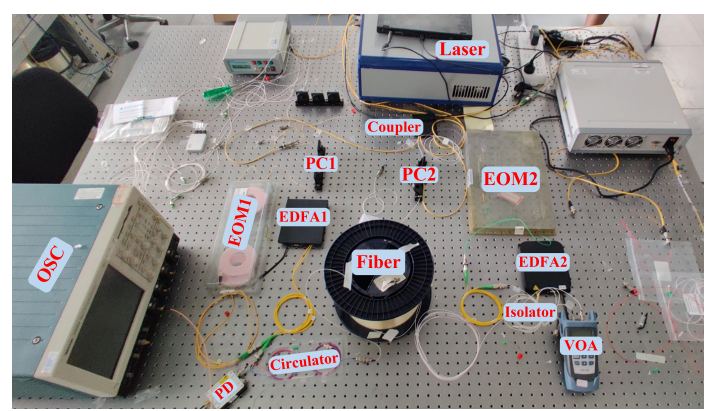

(b)

Figure 1. Brillouin optical time domain analysis (BOTDA) distributed sensing system, (a) schematic diagram, and (b) physical diagram.

When the system has a $500 \mathrm{MHz}$ sampling rate, 16 times average, and a frequency scanning range of $200 \mathrm{MHz}$ with a frequency step of $1 \mathrm{MHz}$ are adopted, the raw 3D BGS image containing is obtained. In this BGS image, the horizontal axis coordinate $x$ represents the position of sensing optical fiber, and perpendicular axis coordinate $y$ denotes pumpprobe frequency $\Delta v$, while axis perpendicular to horizontal plane represents Brillouin gain. The object processed is 2D image (shown in Figure 2) containing $200 \times 100,000$ pixels, which can be acquired by mapping position-frequency pair $(z, \Delta v)$ to pixel point $(x, y)$ and color value representing gain $g$. A simple visual inspection of the raw BGS images indicates that the details of BGS profile is almost covered with white noise points even it is averaged 16 times to eliminate random noise. Especially at the end of the FUT, the abrupt change of temperature in the heating zone cannot be observed. Therefore, some image denoising techniques are introduced to process the raw BGS data. 


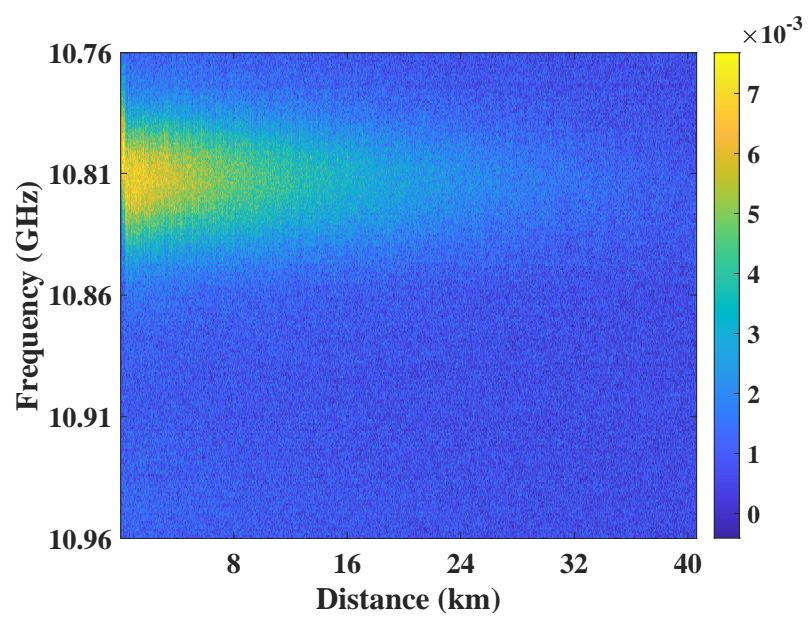

Figure 2. 2D Brillouin gain spectrum.

\subsection{GS Image Denoising Algorithm}

The main noise of BGS image is additive Gaussian white noise. As a classical filtering algorithm, Gaussian filter is a kind of linear smoothing filter, which is suitable for eliminating Gaussian noise and shot noise, and is widely used in image processing. Gaussian filter denoising is used to obtain a weighted average of the pixel value of the whole image. Each pixel value is obtained by the weighted average of its own value and other pixel values in the neighborhood. A Gaussian filter can be implemented by following steps: First, a rectangular neighborhood around the pixel to be processed is selected to form a sliding window; second, a two-dimensional Gaussian distribution function is constructed on this neighborhood; third, the gray values of the pixels in the neighborhood is convoluted with this two-dimensional Gaussian function and the convolution result is used as the gray value of the selected point; and finally, the sliding window is moved to the next pixel the same operation is performed, and all pixels in the image area are traversed.

\subsection{Non-Local Means Image Denoising Algorithm}

NLM image filtering algorithm is proposed by Buades in 2008 [16,17]. The algorithm model extends from local, semi-local to nonlocal by taking advantage of redundancy of natural images. Therefore, NLM image denoising technique can effectively filter out various random noises. The noise signal being assumed to be additive white Gaussian noise, an image with noise $(v)$ can be expressed as

$$
v(i)=x(i)+n(i),
$$

where $x$ is original image, $n$ represents Gaussian noise, and $i$ denotes pixel position. The basic idea of NLM is that the target pixel is not only related to its neighborhood pixels, but also related to other pixels in the whole image. The current pixel value is obtained by the weighted average of all pixels with similar structure in the image. The weight of each pixel is calculated by the Gaussian weighted Euclidean distance between the image block centered on it and the image block centered on the current pixel. The image with noise $(v(i))$ filtered by NLM can be expressed by

$$
\operatorname{NLM}(v(i))=\frac{1}{\sum_{j} \omega(i, j)} \sum_{j \in I} \omega(i, j) v(i),
$$

where I denotes pixel set of image $v$ and $\omega(i, j)$ is the weighted kernel function which can be determined by the similarity between pixel $i$ and $j$. If $N_{i}$ and $N_{j}$ are used to represent the square neighborhood centered on pixel $i$ and $j$, respectively, the similarity between 
pixel $i$ and $j$ depends on the similarity of neighborhood matrix $v\left(N_{i}\right)$ and $v\left(N_{j}\right)$, and can be expressed as

$$
\omega(i, j)=\exp \left(\frac{\left\|v\left(N_{i}\right)-v\left(N_{j}\right)\right\|_{2, a}^{2}}{h^{2}}\right),
$$

where $\left\|v\left(N_{i}\right)-v\left(N_{j}\right)\right\|_{2, a}^{2}$ represents the Gaussian weighted Euclidean distance, $a$ is the standard deviation of Gaussian kernel, and $h$ denotes smoothing coefficient which can determined by noise intensity. According to Equation (2), the establishment of kernel function $\omega(i, j)$ is a key to NLM filtering algorithm, which directly determines the noise reduction ability of NLM algorithm. $\omega(i, j)$ is independent of the geometry and only depends on the similarity of the data around pixels $i$ and $j$. This characteristic determines that the method is nonlocal. The degree of similitude and redundancy in the data is evaluated in a patch-by-patch basis by calculating the Euclidean distance. Two windows with a high level of similitude have small Euclidean distance and can be averaged to reduce noise. In our scheme, the similarity window has been chosen of size $3 \times 3$ to ensure that the processing has not any detrimental impact on the real spatial resolution of the sensor. On the other hand, the optimum value of smoothing control parameter $h$, which determines the level of blurring, depends on the noise level of the data.

\subsection{Quaternion Wavelet Transform Image Denoising Algorithm}

As a new kind of multi-resolution analysis tool of image processing, QWT is approximate shift-invariant. In order to use QWT image denoising technique, the image decomposition based on QWT must be performed, and its schematic is shown in Figure 3. The L sub-band or H sub-band is obtained by 1D discrete WT (DWT) according to the row of original image, then one low-frequency sub-band (LL), three horizontal (HL), vertical $(\mathrm{LH})$, and diagonal $(\mathrm{HH})$ sub-bands are formed by the column of $\mathrm{L}$ and $\mathrm{H}$ sub-band according to 1D DWT. The coefficient for four sub-bands is expressed by real function $f(x, y)$.

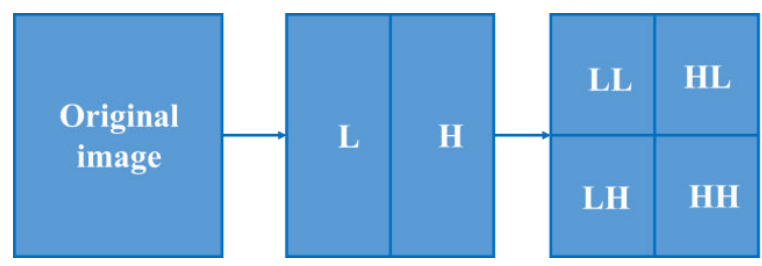

Figure 3. Image wavelet decomposition schematic diagram.

On this basis, the QWT adopts four real DWT. The first real discrete wavelet $f(x, y)$ corresponds to the real part of quaternion wavelet, the second and third real discrete wavelet are obtained by the partial Hilbert transformation along the $X$-axis and $Y$-axis $\left(H_{H x}, H_{H y}\right)$, and the last one is obtained by the whole Hilbert transform $\left(H_{H x y}\right)$. The last three parts correspond to the three imaginary parts of quaternion wavelet, respectively. Therefore, QWT for an image is expressed by

$$
f^{q}(x, y)=f(x, y)+i H_{H x}[f(x, y)]+j H_{H y}[f(x, y)]+k H_{H x y}[f(x, y)] .
$$

Wavelet decomposition can be continued according to the route described above. In order to select the level of multi-level quaternion wavelet, the denoising effect, and computational complexity, and degree of time-consuming behavior should be considered. For the raw BGS image shown in Figure 2, when the decomposition layers number three and four, the denoising performance is the best. With increasing decomposition layers, the image reflected by the low-frequency coefficients of QWT will be smoother, and more edge details will be decomposed to high-frequency coefficients. Considering the algorithm complexity and denoising performance, the layers of decomposition is set to three [18]. 
Quaternion $f^{q}(x, y)$ also can be written by another form [19]:

$$
f^{q}(x, y)=\left|f^{q}(x, y)\right| e^{i \varphi} e^{j \theta} e^{k \psi},
$$

where $\left|f^{q}(x, y)\right|$ is the modulus of $f^{q}(x, y), \varphi, \theta, \psi$ are three phase angles which are uniquely defined within the range $(\varphi, \theta, \psi) \in[\pi, \pi] \times[-\pi / 2, \pi / 2] \times[-\pi / 4, \pi / 4]$. The quaternion wavelet $f^{q}(x, y)$ corresponds to one amplitude and three phases which can be depicted to four images shown in Figure 4. The results imply that three phase images mainly reflect the edge and texture information of the image, which are randomly and disorderly distributed to the noise, while the amplitude image is greatly affected by the noise. Therefore, the principle of denoising is that the amplitude coefficients are processed according to certain criterion and the phase coefficients remain. For the amplitude image, most of the energy is concentrated on a small number of coefficients with large amplitude, which represent the edge and other details. The noise mainly corresponds to the coefficients with smaller amplitude. It is reasonable to set a certain threshold to reduce the noise in amplitude image. The following steps are performed to remove amplitude noise. First, the variance of noise according to robust estimation method is calculated by [20]

$$
\sigma_{n}=\frac{\operatorname{Median}\left(\left|\omega_{i, j}\right|\right)}{0.6745},
$$

where $\omega_{i, j}$ is the finest sub-band coefficient and $\left|\omega_{i, j}\right|$ denotes its magnitude.

Then, the amplitude variance $\sigma_{w}^{2}$ is estimated by

$$
\sigma_{w}^{2}=\frac{1}{N^{2}} \sum_{\left|\omega_{j}\right| \in W(k)}\left(\left|\omega_{j}\right|\right)^{2},
$$

where $W(k)$ is the square-shaped neighborhood window of $\left|\omega_{j}\right|$ and $N^{2}$ is the size of the window. Therefore, QWT coefficients can be obtained by

$$
w=m+n,
$$

where $w, m$, and $n$ are matrixes of the observed QWT coefficients, ideal QWT coefficients, and noise QWT coefficients, respectively. The variance, $\sigma_{m}$, can be estimated by

$$
\sigma_{m}=\sqrt{\max \left(\sigma_{w}^{2}-\sigma_{n}^{2}, 0\right)}
$$

Third, a shrink threshold needs to determined. For each sub-band in QWT domain, if the threshold is too low, the denoising effect is not obvious. If the threshold is too high, more details will be "killed". Therefore, a reasonable noise-free coefficient's magnitude $m$ is required by using the Bayes shrink threshold $T$ [21]

$$
T=\frac{\sigma_{n}^{2}}{\sigma_{m}} .
$$

Fourth, the element of coefficients matrix $m$ can be determined by soft thresholding function

$$
m_{k, j}^{i}=\left\{\begin{array}{cl}
\operatorname{sign}\left(w_{k, j}^{i}\right) \times\left(\left|w_{k, j}^{i}\right|-T_{k, j}^{i}\right), & \text { if }\left|w_{k, j}^{i}\right|>T_{k, j}^{i}, \\
0, & \text { else }
\end{array}\right.
$$

where $w_{k, j}^{i}$ is the $i$ th coefficient in the $j$ direction of $k$ layer, $T_{k, j}^{i}$ is the corresponding threshold, and $m_{k, j}^{i}$ is coefficient after denoising. 

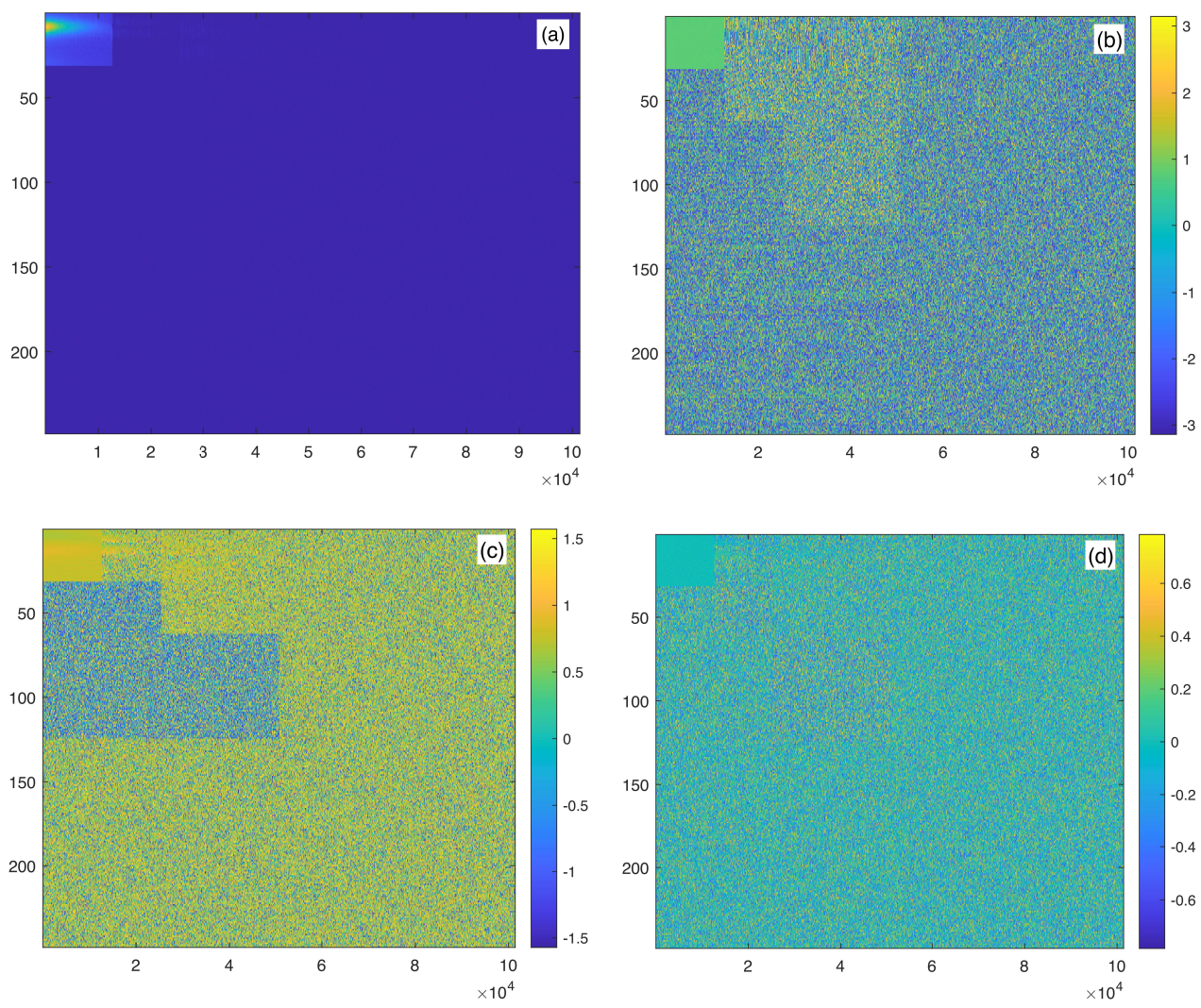

Figure 4. Results of decomposition by QWT (a) magnitude, and (b-d) 3 terms of phase $(\phi, \theta, \varphi)$.

Finally, by performing inverse QWT on the estimated magnitude and the original phase to get QWT coefficients, the denoised BGS image can be reconstructed.

From the description of the algorithm principle of the three image denoising methods above, we can see that the GS algorithm is a local average method, and its denoising effect is related to the size of the selected rectangular window, while the kernel function of NLM depends on the similarity of the data around the selected pixel and pixels of its neighborhood and that the weighted average window size is dynamically adjusted with the similarity. Compared with GS, the NLM algorithm can highlight the texture and edge information of the image. However, Gaussian filtering and NLM algorithms belong to image smoothing denoising methods, and image blurring is inevitable. For BGS information extraction, image blurring will lead to the reduction in spatial resolution. In the QWT image denoising algorithm, after the original image being decomposed by QWT, the main texture and edge information are concentrated in three phase images, while the high-frequency noise mainly in the amplitude image. The effective separation of the useful information and noise is achieved from the source. Therefore, compared with other local or non local smoothing denoising algorithms, QWT image denoising algorithm can achieve better filtering effect and higher spatial resolution. Due to its local smooth feature, GS denoising has high time efficiency. NLM image denoising needs to consider the similarity of the whole image, so it has high computational complexity and lower time efficiency. QWT image denoising algorithm only needs three layers of wavelet transform, soft threshold function denoising processing, and inverse QWT. Its computational complexity is lower than NLM algorithm, so it has higher time efficiency.

\section{Discussion}

For the convenience of comparison, the processing results of original data, and denoised data by GS, NLM, and QWT is shown in Figures 5-8, respectively. In order to present the denoising effect visually, BGS data can be mapped into an 3D image. The coordinate pixel point $(x, y)$ in horizontal plane represents the position-frequency pair $(z, \Delta v)$, 
while the axis perpendicular to horizontal plane represents the Brillouin gain. In Figure 5a, the whole 3D BGS image mapped by raw BGS data is covered in noise, so that the BGS drift to high frequency can hardly be detected in the heating region at the end of sensing fiber. Therefore, it is necessary to denoise the raw BGS data. Figure 6a shows the 3D BGS image by using GS image denoising algorithm. Considering that the size of the original image to be processed is $200 \times 100,000$, the sliding window is a rectangular window with a size of $20 \times 200$. The standard deviation of the image is estimated as $8.23 \times 10^{-2}$. The results show that some details of image are revealed after GS denoising. In the high-temperature region at the end of sensing fiber, the drift of BGS to high frequency can be observed clearly. The residual noise can hardly be observed. GS achieves a very good denoising effect because 20 points are smoothed in the frequency domain and 200 points are smoothed in the spatial domain. However, frequency domain smoothing will reduce the BFS sensitivity, while the spatial domain will reduce its spatial resolution. Figure 7a is the 3D BGS image using NLM image denoising algorithm. The similarity window has been chosen of size $10 \times 10$ to ensure that the processing has not detrimental impact on the real spatial resolution of the sensor. The smoothing control parameter $h$ is set to ten times the noise standard deviation, namely, $8.23 \times 10^{-3}$. The results express that the noise in raw BGS image was almost completely cleared and the details of the image have been highlighted clearly. The drift of BGS to high frequency at the end of FUT is easy to find. Figure 8a is the 3D BGS image using QWT image denoising algorithm. Comparing to 3D images from NLM and QWT denoising algorithm, two BGS images are almost exactly the same, with the noise being cleared up and the drift of BGS to high frequency from temperature jump is obvious.
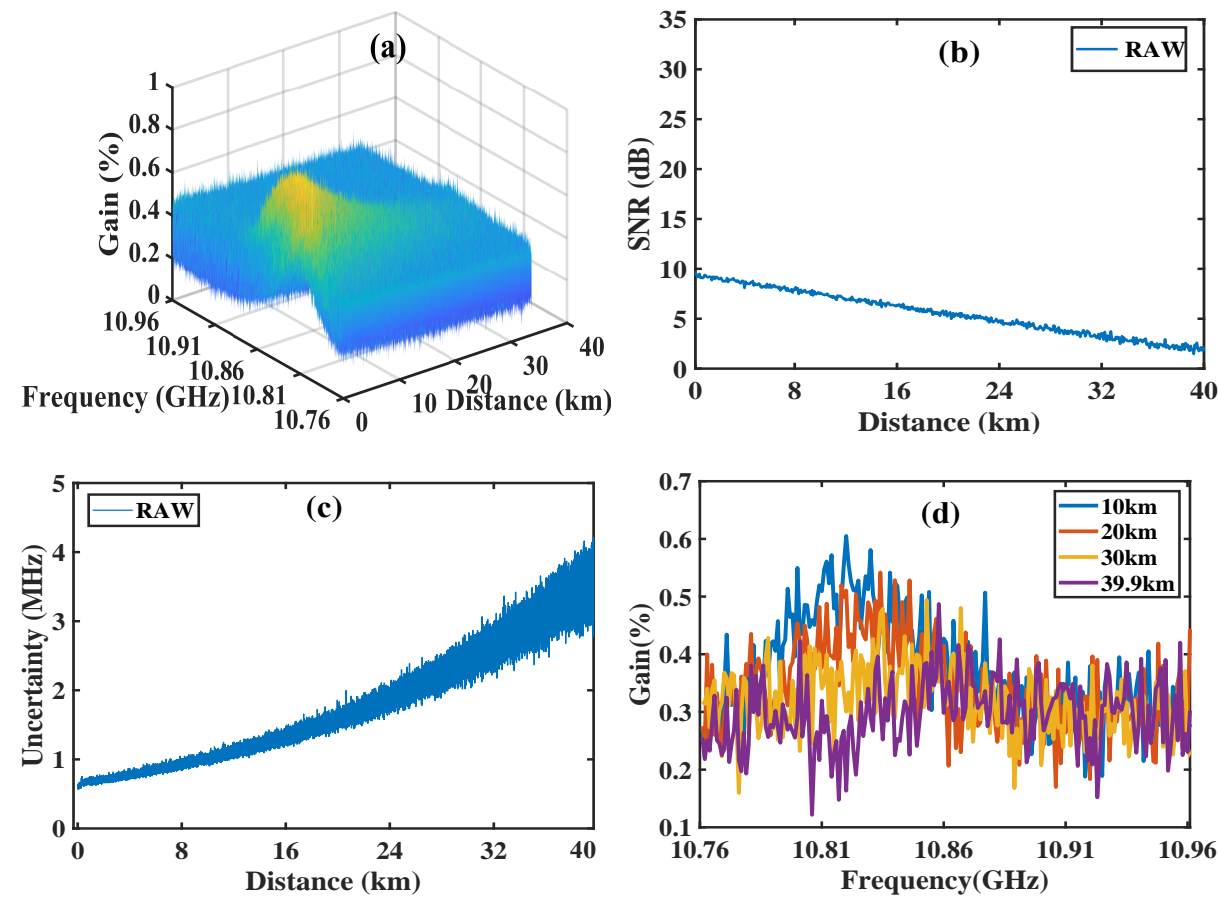

Figure 5. (a) Measured Brillouin gain spectrum (BGS) distribution along fiber under test (FUT) without denoising, corresponding signal-to-noise ratio (SNR) (b), uncertainty frequency (c), and extracted BGS curves (d). 
(a)

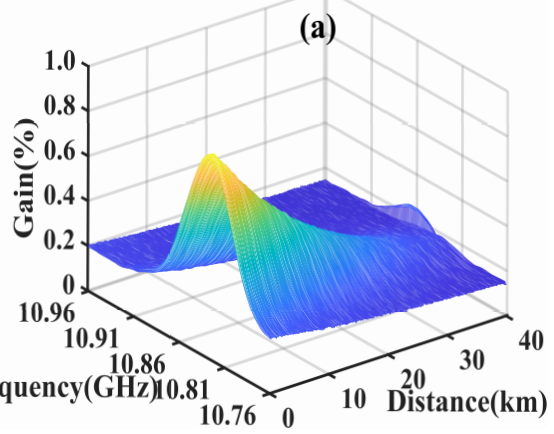

$10.76 \quad 0 \quad 10$ Distance $(\mathrm{km})$

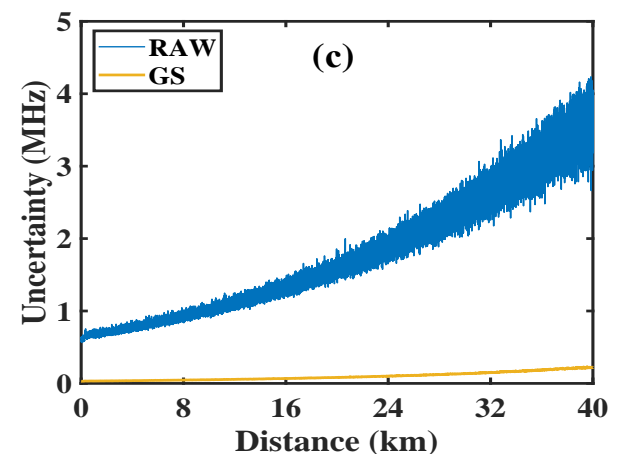

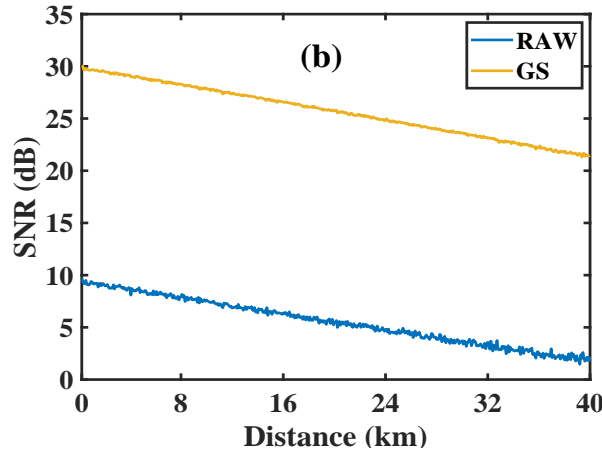

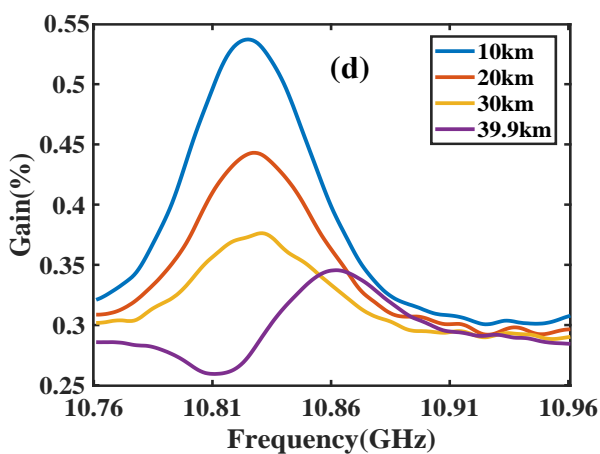

Figure 6. (a) BGS distribution from BGS data processed by GS denoising algorithm, corresponding SNR (b), uncertainty frequency (c), and extracted BGS curves (d).
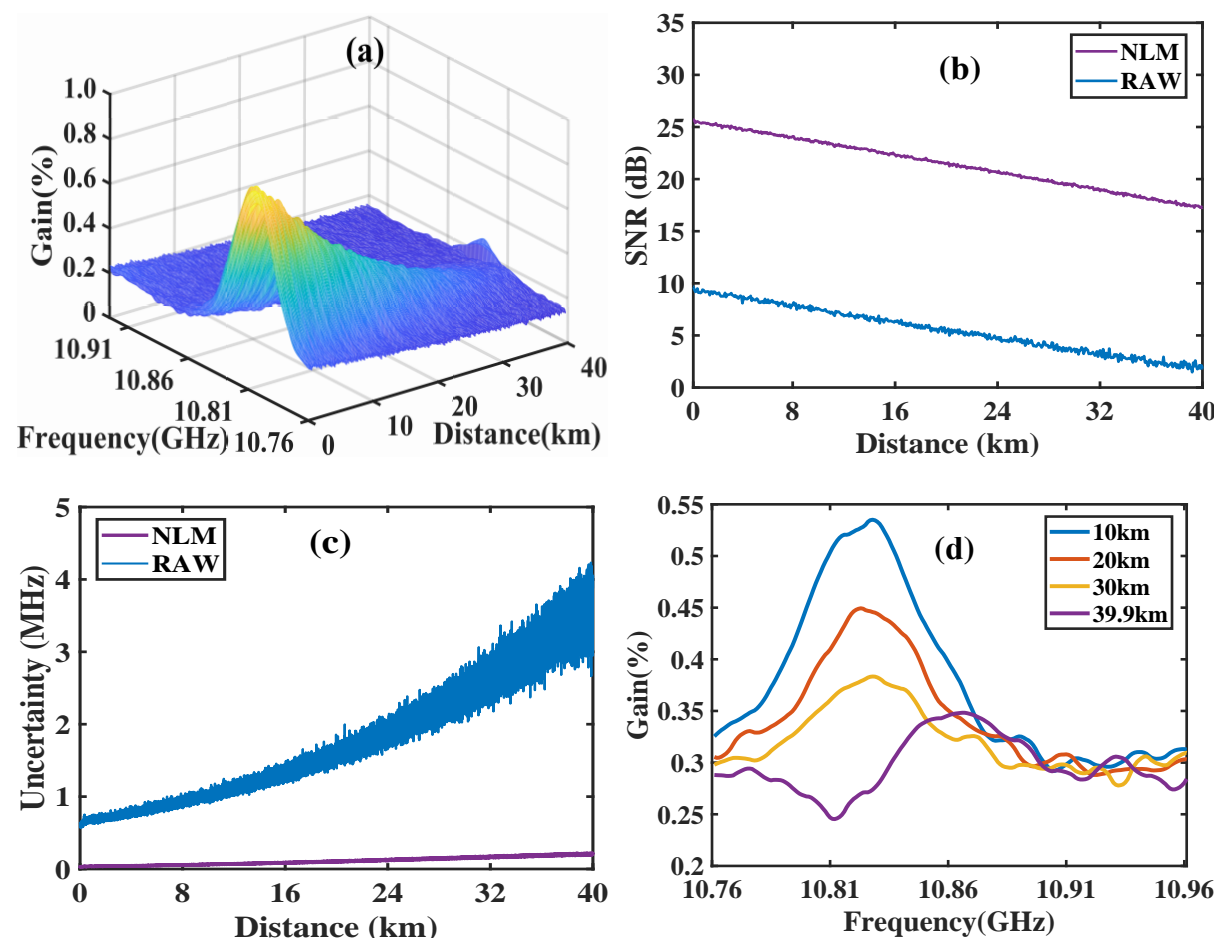

Figure 7. (a) BGS distribution from BGS data processed by NLM denoising algorithm, corresponding SNR (b), uncertainty frequency (c), and extracted BGS curves (d).

The denoising effect of various algorithms can be quantitatively evaluated by the signal-to-noise ratio of the image. The signal amplitude comes from the peak Brillouin gain in the NGS image. For the region without Brillouin gain at the end of each scanning frequency, we calculate the corresponding standard deviation, and then take the mean value of these standard deviations as the noise level of the system [7]. The SNR curves 
along FUT form raw image, and the images by using GS, NLM, and QWT image denoising algorithms are shown in Figures $5 b, 6 b, 7 b$ and $8 b$. For the raw BGS image, the SNR of the front of FUT is $9.69 \mathrm{~dB}$, while approximately $2.14 \mathrm{~dB}$ at the end of $40 \mathrm{~km}$ long sensing fiber. After GS denoising, the SNR at the end of sensing fiber is surprisingly increased to $21.38 \mathrm{~dB}$. The results in Figures $7 \mathrm{~b}$ and $8 \mathrm{~b}$ show that the SNR at the end of sensing fiber is improved from $2.14 \mathrm{~dB}$ to $17.42 \mathrm{~dB}$ by using NLM denoising, and $17.26 \mathrm{~dB}$ by using QWT denoising. Therefore, only considering the effect of noise removal, NLM, and QWT are almost equivalent and have some disadvantages over GS.

Another parameter to evaluate the denoising effect is the frequency uncertainty $\sigma_{z}$, which reflects the stability of the output and can be estimated by [22]

$$
\sigma_{z}=\frac{\hat{\sigma}}{g(z)} \sqrt{\frac{3}{4} \delta \times \Delta v_{B}}
$$

where $g(z)$ is the Brillouin gain in position $z, \delta$ is the scanning frequency interval of the probe light, and $\Delta v_{B}$ represents full width at half maximum (FWHM) of corresponding BGS curve. The distribution curves of frequency uncertainty form raw image, and the images processed by GS, NLM, and QWT are shown in Figures $5 c, 6 c, 7 c$ and $8 c$. The results indicate that the frequency uncertainty is approximately $0.73 \mathrm{MHz}$ from raw BGS data at the front of FUT and approximately $4.13 \mathrm{MHz}$ at the end. After being denoised by GS, frequency uncertainty decreases to $0.03 \mathrm{MHz}$ at the front of FUT and $0.21 \mathrm{MHz}$ at the end. The frequency uncertainty is cut down to 0.04 and $0.05 \mathrm{MHz}$ at the front of FUT, and $0.21 \mathrm{MHz}$ and $0.24 \mathrm{MHz}$ at the end by using NLM and QWT denoising methods, respectively. In the aspect of frequency uncertainty, the QWT is equivalent to NLM and has obvious disadvantages over GS.
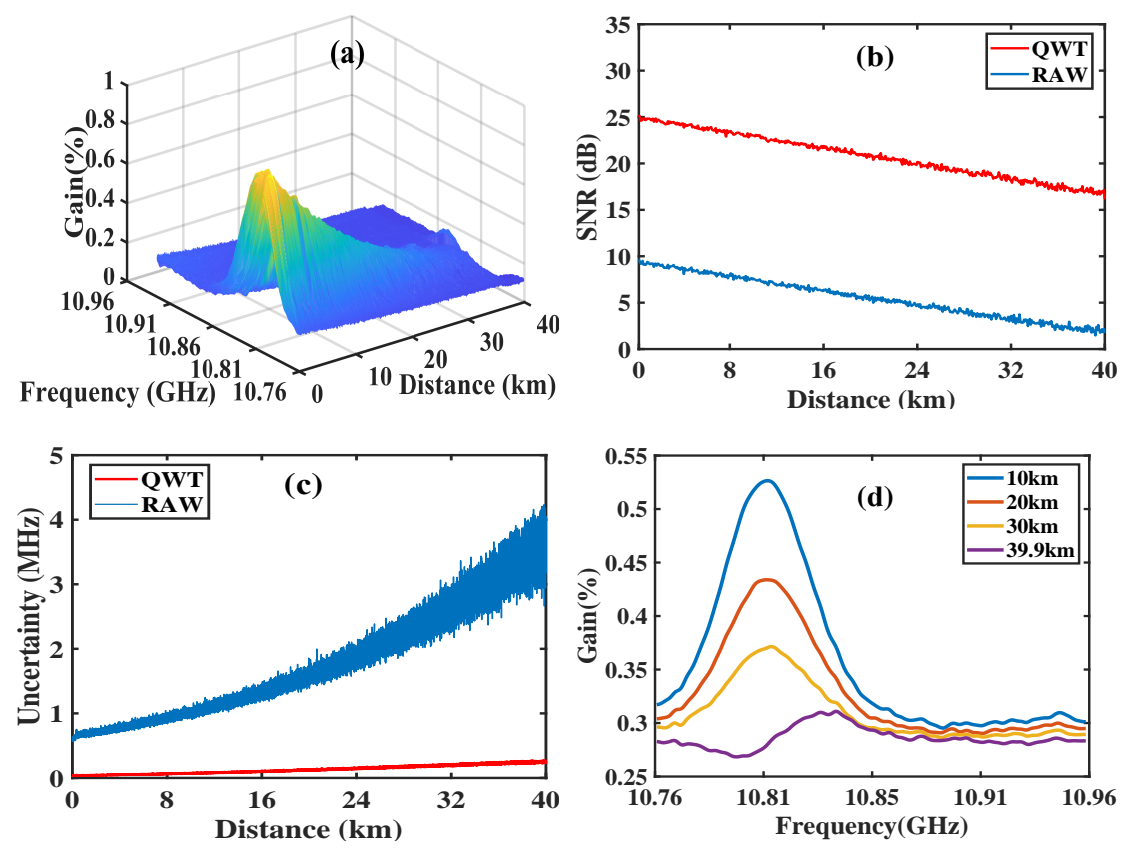

Figure 8. (a) BGS distribution from BGS data processed by QWT denoising algorithm, corresponding SNR (b) uncertainty frequency (c), and extracted BGS curves (d).

The accuracy of extracted BFS or temperature also depends on the denoising effect of BGS data. We chose BGS curves at $1 \mathrm{~km}, 13 \mathrm{~km}, 26 \mathrm{~km}$, and $39.9 \mathrm{~km}$ as the research objects, and extract the corresponding BFS values by Lorentz curve fitting (LCF) method. The extracted BGS curves from raw image are shown in Figure 5d. Even after 16 times of time-domain averaging, all the curves are full of all kinds of "burrs". The BFS values extracted from the BGS curves at $1 \mathrm{~km}, 13 \mathrm{~km}$, and $26 \mathrm{~km}$ of the sensing fiber at room 
temperature are $10.828 \mathrm{GHz}, 10.834 \mathrm{GHz}$, and $10.831 \mathrm{GHz}$, respectively, corresponding to $28.1^{\circ} \mathrm{C}, 35.3^{\circ} \mathrm{C}$, and $31.2^{\circ} \mathrm{C}$, which deviate from actual room temperature of $24^{\circ} \mathrm{C}$, while the BFS value extracted from the heating zone is $10.866 \mathrm{GHz}$, corresponding to $67.1^{\circ} \mathrm{C}$, which deviates from actual temperature of $64^{\circ} \mathrm{C}$ suffered by sensing fiber. The results indicate that it is difficult to extract accurate BFS or temperature from raw BGS data. The BGS curves from the image denoised by GS are shown in Figure 6d. All curves is very smooth without any "burrs" indicate that GS with $20 \times 100$ sliding window completely eliminate the noise of BGS image. The BFSs at $1 \mathrm{~km}, 13 \mathrm{~km}$, and $26 \mathrm{~km}$ are $10.8240 \mathrm{GHz}$, 10.8242 GHz, and $10.8243 \mathrm{GHz}$, corresponding to $24^{\circ} \mathrm{C}, 24.2{ }^{\circ} \mathrm{C}$ and $24.3^{\circ} \mathrm{C}$. The BFS of $10.863 \mathrm{GHz}$ is obtained at $39.9 \mathrm{~km}$ of sensing fiber, corresponding to $63.97{ }^{\circ} \mathrm{C}$, which is very close to the actual temperature at heating zone. Figure $7 \mathrm{~d}$ shows BGS curves from the image denoised by NLM. All the curves are very smooth without any "burrs", but the BGS curve has been deformed and no longer retains Lorentz shape. From the denoising effect, NLM is an ideal algorithm. The BFSs of the sampling points at room temperature zone are 10.8236 GHz, $10.8230 \mathrm{GHz}$, and $10.8246 \mathrm{GHz}$, corresponding to $23.6{ }^{\circ} \mathrm{C}, 23^{\circ} \mathrm{C}$, and $24.6{ }^{\circ} \mathrm{C}$. The BFS at $39.9 \mathrm{~km}$ is $10.8633 \mathrm{GHz}$, corresponding to $64.3^{\circ} \mathrm{C}$. The calculated temperature values are consistent with the actual temperature suffered by sensing fiber. Figure $8 \mathrm{~d}$ shows BGS curves from image processed by QWT denoising algorithm. Although there are scattered small "burrs" on the curves, all curves keep Lorentz shape. The BFS values of the four research points are $10.824 \mathrm{GHz}, 10.8241 \mathrm{GHz}, 10.824 \mathrm{GHz}$, and $10.8631 \mathrm{GHz}$, corresponding to $24^{\circ} \mathrm{C}, 24.1^{\circ} \mathrm{C}, 24^{\circ} \mathrm{C}$, and $64.1^{\circ} \mathrm{C}$. From the above results, we can see that QWT denoising algorithm can achieve more accurate BFS or temperature values than that from BGS images denoised by using NLM algorithm, and is are at the same level as GS algorithm.

We select the BFS distribution of $800 \mathrm{~m}$ at the end of FUT to investigate the accuracy and spatial resolution of BFS obtained by raw data, GS, NLM, and QWT denoising algorithms, and corresponding results are shown in Figure 9. The results indicate that the BFS distributions fluctuate within $\pm 2.5 \mathrm{MHz}$ under the condition of the actual temperature $44^{\circ} \mathrm{C}, 55^{\circ} \mathrm{C}, 64^{\circ} \mathrm{C}$, and $75^{\circ} \mathrm{C}$ when BFSs are retrieved by LCF with raw BGS data. The details of the temperature transition region inserted in Figure 9 a show the spatial resolution is about $3 \mathrm{~m}$, which is consistent with the time domain width of the pump laser pulse. Although GS significantly reduces the fluctuation range of BFS to about $0.2 \mathrm{MHz}$, the spatial resolution also decreases dramatically. According to Figure 9b, the length of temperature transition region exceeds $80 \mathrm{~m}$ when 100 points in the spatial domain are used for smoothing. Although the spatial resolution can be improved by reducing the size of the smoothing window, it is accompanied by a sharp deterioration of SNR. BFS distributions from BGS images by using NLM denoising algorithm are shown in Figure 9c. BFS distributions are in agreement with the corresponding temperatures exerted in the last $200 \mathrm{~m}$ fiber within $\pm 0.2 \mathrm{MHz}$ accuracy and the spatial resolutions from NLM are about $8 \mathrm{~m}$. From the BFS distributions from BGS images denoised by QWT algorithm, BFS accuracy of $0.2 \mathrm{MHz}$ can be achieved, and the BFS values are consist with actual temperature $44^{\circ} \mathrm{C}$, $55^{\circ} \mathrm{C}, 64{ }^{\circ} \mathrm{C}$, and $75^{\circ} \mathrm{C}$ suffered by the sensing fiber. The details in Figure $9 \mathrm{~d}$ show that the length of the transition section is $3 \mathrm{~m}$ when the temperature distribution along the sensing fiber has an abrupt variation. From the above discussion, we can conclude that the QWT denoising algorithms have similar detection accuracy better than GS, GS, and NLM algorithms can bring in the degrade of the spatial resolution, while QWT denoising algorithm is with the highest spatial resolution.

The time efficiency of the denoising algorithm depends on the computational complexity of the algorithm and is measured by the execution time of the algorithm in the computer. Due to its low computational complexity, it takes no more than $1 \mathrm{~s}$ to process a BGS image with $200 \times 100,000$ sampling points by using GS algorithm when $10 \times 100$ smoothing window is adopted, while the NLM algorithm needs more than 40 min because it involves correlation calculation. For the same BGS image, it takes only $32 \mathrm{~s}$ to implement the QWT denoising algorithm with 3-layer QWT image decomposition, which can meet 
the time requirements of many large-scale infrastructures structure health monitoring. In addition, by increasing the scan frequency interval or decreasing the sampling rate of ADC, the running time of QWT algorithm can be shortened, and the accuracy and spatial resolution of BFS are acceptable for structure health monitoring. Considering the denoising effect, detection accuracy, spatial resolution, and execution efficiency, as per Table 1, the QWT image denoising algorithm has certain advantages over GS and NLM.
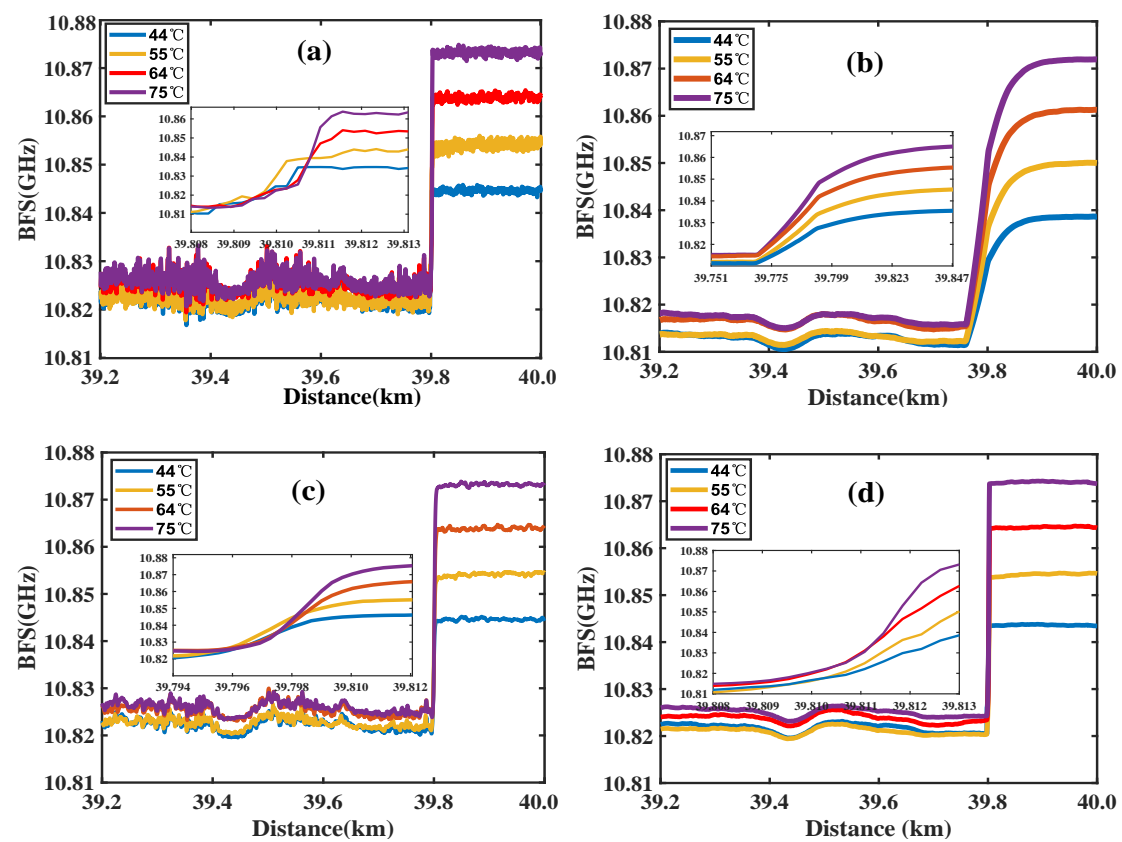

Figure 9. BFS distribution from raw data (a) and from BGS image denoised by GS (b), NLM (c) and QWT (d).

Table 1. Experimental results and comparison with systems processed by other algorithms.

\begin{tabular}{ccccc}
\hline & Raw Data & GS & QWT & NLM \\
\hline $\begin{array}{c}\text { SNR } \\
\text { Uncertainty at } \\
\text { front }\end{array}$ & $9.6 \mathrm{~dB}$ & $21.38 \mathrm{~dB}$ & $17.26 \mathrm{~dB}$ & $17.42 \mathrm{~dB}$ \\
$\begin{array}{c}\text { Uncertainty at } \\
\text { end }\end{array}$ & $0.73 \mathrm{MHz}$ & $0.03 \mathrm{MHz}$ & $0.05 \mathrm{MHz}$ & $0.04 \mathrm{MHz}$ \\
$\begin{array}{c}\text { Spatial } \\
\text { Resolution }\end{array}$ & $3.13 \mathrm{MHz}$ & $0.21 \mathrm{MHz}$ & $0.24 \mathrm{MHz}$ & $0.21 \mathrm{MHz}$ \\
Running time & $1 \mathrm{~m}$ & $80 \mathrm{~m}$ & $3 \mathrm{~m}$ & $8 \mathrm{~m}$ \\
\hline
\end{tabular}

\section{Conclusions}

In this paper, we describe in detail the principles of GS, NLM, and QWT image denoising algorithms, and propose these algorithms to denoise BGS images. From the principle analysis, the QWT algorithm effectively separates the noise and useful information from the source, and retains more image details and is an efficient image denoising algorithm. A BOTDA experimental system is set up. BGS data are collected and processed by GS, NLM, and QWT image denoising algorithms. The GS algorithm has high time efficiency and good measurement accuracy, but with poor spatial resolution. After being denoised by using NLM algorithm, at the end of FUT, SNR increases from $2.14 \mathrm{~dB}$ to $16.68 \mathrm{~dB}$ and frequency uncertainty decreases from $4.13 \mathrm{MHz}$ to $0.19 \mathrm{MHz}$. The BFS accuracy is kept in the range of $\pm 0.2 \mathrm{MHz}$. It takes more than $40 \mathrm{~min}$ for a computer to process a BGS image with $400 \times 100,000$ sampling points by using NLM denoising algorithm. By using QWT denoising algorithm, SNR increases to $17.26 \mathrm{~dB}$ and frequency uncertainty decreases to $0.24 \mathrm{MHz}$. The results show that QWT and NLM algorithms have the same denoising 
effect, but QWT algorithm can achieve nearly perfect Lorentz curve, while NLM causes BGS curve deformation. Therefore, QWT algorithm can get higher detection accuracy. The QWT algorithm can achieve higher spatial resolution because smoothing is not required. In addition, it takes less than $32 \mathrm{~s}$ to denoise the same BGS image and $3 \mathrm{~m}$ spatial resolution can achieved. In conclusion, by comparing the denoising effect, detection accuracy, spatial resolution, and execution efficiency, the QWT denoising algorithm is suitable for BGS data processing in BOTDA. We believe that the research results of this paper can promote the application of the QWT image denoising technique in distributed optical fiber sensing, even in large infrastructure structure health monitoring systems. Moreover, structural health monitoring includes not only temperature, but also tension, pressure, vibration, and so on. Our future work will focus on real-time and accurate extraction of these other parameters to provide assistance for the development of industrial Internet of Things.

Author Contributions: Formal analysis, H.W. (Haoyu Wei) and C.L.; Investigation, Q.W., X.L., H.W. (Hongxin Wu) and L.F.; Methodology, Y.W.; Supervision, X.X.; Writing-original draft, Y.W.; Writing-review and editing, Y.W. All authors have read and agreed to the published version of the manuscript.

Funding: This work is supported by the Research Innovation Fund for College Students of Beijing University of Posts and Telecommunication (202002028), the National Natural Science Foundation of China (61675030), and the National Key Research and Development Program of China (2018YFB1801200).

Acknowledgments: We thank all the supporters who participated in the research.

Conflicts of Interest: The authors declare no conflict of interest.

$\begin{array}{ll}\begin{array}{l}\text { Abbreviations } \\ \text { The following abbreviations are used in this man }\end{array} \\ \text { GS } & \text { Gaussian smoothing } \\ \text { NLM } & \text { non-local means } \\ \text { QWT } & \text { Quaternion Wavelet Transform } \\ \text { BGS } & \text { Brillouin gain spectrum } \\ \text { BOTDA } & \text { Brillouin optical time domain analysis } \\ \text { SNR } & \text { signal to noise ratio } \\ \text { BFS } & \text { Brillouin frequency shift } \\ \text { WD } & \text { wavelet denoising } \\ \text { LWT } & \text { lifting wavelet transform } \\ \text { 2D } & \text { 2-dimensional } \\ \text { 2D-WD } & \text { 2D wavelet denoising } \\ \text { BM3D } & \text { block matching 3D } \\ \text { CNN } & \text { convolutional neural network } \\ \text { FUT } & \text { fiber under test } \\ \text { CW } & \text { continuous wave } \\ \text { EOM1 } & \text { Electro-optic modulator 1 } \\ \text { PPG } & \text { programmable pattern generator } \\ \text { EDFA1 } & \text { erbium-doped fiber amplifier 1 } \\ \text { VOA } & \text { variable optical attenuator } \\ \text { BPF } & \text { band-pass filter } \\ \text { OSC } & \text { oscilloscope } \\ \text { DWT } & \text { discrete WT } \\ \text { LCF } & \text { Lorentz curve fitting } \\ & \end{array}$

\section{References}

1. Alahbabi, M.; Cho, Y.T.; Newson, T.P. Comparison of the methods for discriminating temperature and strain in spontaneous Brillouin-based distributed sensors. Opt. Lett. 2004, 29, 26-28. [CrossRef] [PubMed]

2. Wang, B.; Wang, L.; Guo, N.; Zhao, Z.; Yu, C.; Lu, C. Deep neural networks assisted BOTDA for simultaneous temperature and strain measurement with enhanced accuracy. Opt. Express 2019, 27, 2530-2543. [CrossRef] [PubMed] 
3. Li, Z.; Yan, L.; Zhang, X.; Pan, W. Temperature and Strain Discrimination in BOTDA Fiber Sensor by Utilizing Dispersion Compensating Fiber. IEEE Sens. J. 2018, 18, 7100-7105. [CrossRef]

4. Lee, C.C.; Chiang, P.W.; Chi, S. Utilization of a dispersion-shifted fiber for simultaneous measurement of distributed strain and temperature through Brillouin frequency shift. IEEE Photonics Technol. Lett. 2001, 13, 1094-1096. [CrossRef]

5. Li, Z.; Yan, L.; Shao, L.; Wei, P.; Zhang, Y. Precise Brillouin gain and phase spectra measurements in coherent BOTDA sensor with phase fluctuation cancellation. Opt. Express 2016, 24, 4824-4833. [CrossRef] [PubMed]

6. Shimizu, S.; Kitamura, S.; Narieda, S.; Naruse, H. Denoising of Brillouin gain spectrum via dictionary learning considering strain variation. IEICE Tech. 2020, 119, 29-34.

7. Fujimoto, T.; Naruse, H.; Nishino, T. Noise reduction of Brillouin gain spectrum using non-negative matrix factorization. IEICE Tech. 2018, 117, 37-41.

8. Soto, M.A.; Bolognini, G.; Pasquale, F.D.; Thévenaz, L. Simplex-coded BOTDA fiber sensor with $1 \mathrm{~m}$ spatial resolution over a 50 km range. Opt. Lett. 2010, 35, 259-261. [CrossRef] [PubMed]

9. Liang, H.; Li, W.; Linze, N.; Chen, L.; Bao, X. High-resolution DPP-BOTDA over $50 \mathrm{~km}$ LEAF using return-to-zero coded pulses. Opt. Lett. 2010, 35, 1503-1505. [CrossRef] [PubMed]

10. Wang, F.; Zhu, C.; Cao, C.; Zhang, X. Enhancing the performance of BOTDR based on the combination of FFT technique and complementary coding. Opt. Express 2017, 25, 3504-3513. [CrossRef] [PubMed]

11. Farahani, M.A.; Wylie, M.T.V.; Castillo-Guerra, E.; Colpitts, B.G. Reduction in the Number of Averages Required in BOTDA Sensors Using Wavelet Denoising Techniques. J. Light. Technol. 2012, 30, 1134-1142. [CrossRef]

12. Tangudu, R. Dynamic range enhancement of OTDR using lifting wavelet transform-modified particle swarm optimisation scheme. IET Optoelectron. 2019, 13, 295-302. [CrossRef]

13. Soto, M.A.; Ramirez, J.A.; Thevenaz, L. Intensifying the response of distributed optical fibre sensors using 2D and 3D image restoration. Nat. Commun. 2016, 7, 1-11. [CrossRef] [PubMed]

14. Wu, H.; Wang, L.; Zhao, Z.; Guo, N.; Shu, C.; Lu, C. Brillouin optical time domain analyzer sensors assisted by advanced image denoising techniques. Opt. Express 2018, 26, 5126-5139. [CrossRef] [PubMed]

15. Wu, H.; Wan, Y.; Tang, M.; Chen, Y.; Zhao, C.; Liao, R.; Chang, Y.; Fu, S.; Shum, P.P.; Liu, D. Real-time denoising of Brillouin optical time domain analyzer with high data fidelity using convolutional neural networks. J. Light. Technol. 2018, 37, 2648-2653. [CrossRef]

16. Buades, A.; Coll, B.; Morel, J.M. A non-local algorithm for image denoising. In Proceedings of the 2005 IEEE Computer Society Conference on Computer Vision and Pattern Recognition (CVPR'05), San Diego, CA, USA, 20-25 June 2005; Volume 2, pp. 60-65.

17. Buades, A.; Coll, B.; Morel, J.M. Image denoising methods. A new nonlocal principle. SIAM Rev. 2010, 52, 113-147. [CrossRef]

18. Liu, Y.; Jin, J.; Wang, Q.; Shen, Y.; Dong, X. Region level based multi-focus image fusion using quaternion wavelet and normalized cut. Signal Process. 2014, 97, 9-30. [CrossRef]

19. Bulow, T. Hypercomplex Spectral Signal Representations for the Processing and Analysis of Images. Ph.D. Thesis, ChristianAlbrechts-Universitat zu Kiel, Kiel, Germany, 1999.

20. Donoho, D.L.; Johnstone, J.M. Ideal spatial adaptation by wavelet shrinkage. Biometrika 1994, 81, 425-455. [CrossRef]

21. Chang, S.G.; Yu, B.; Vetterli, M. Adaptive wavelet thresholding for image denoising and compression. IEEE Trans. Image Process. 2000, 9, 1532-1546. [CrossRef] [PubMed]

22. Soto, M.A.; Thévenaz, L. Modeling and evaluating the performance of Brillouin distributed optical fiber sensors. Opt. Express 2013, 21, 31347-31366. [CrossRef] [PubMed] 